\title{
Rheumatoid arthritis synovial fibroblasts promote TREM-1 expression in monocytes via $\mathrm{COX}-2 / \mathrm{PGE}_{2}$ pathway
}

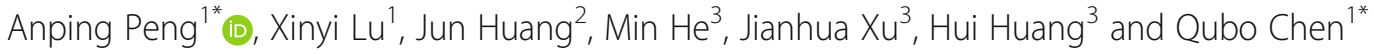

\begin{abstract}
Background: Triggering receptor expressed on myeloid cells-1 (TREM-1) is inducible on monocyte/macrophages and neutrophils and amplifies the inflammatory response. The aim of this study was to determine whether rheumatoid arthritis synovial fibroblasts (RASF) promote the expression of TREM-1 in monocytes and its potential regulatory mechanism.
\end{abstract}

Methods: Synovial fluid and paired peripheral blood from rheumatoid arthritis (RA) patients were analyzed using flow cytometry. Expression of TREM-1 in monocytes was detected after co-culture with RASF, with or without pretreatment with toll-like receptor (TLR) ligands. Whether RASF-regulated TREM-1 level in monocytes require direct cell contact or soluble factors was evaluated by transwell experiment. COX-2 expression and $\mathrm{PGE}_{2}$ secretion in RASF were determined by quantitative PCR ( $(\mathrm{PCR}$ ) and ELISA. RASF, with and without TLR ligand stimulation, were treated with COX-2 inhibitors, COX-2 siRNA (siCOX-2) or EP1-4 antagonists, and the resulting TREM-1 level in CD14 ${ }^{+}$ monocytes was measured using flow cytometry.

Results: TREM-1 was highly expressed in $\mathrm{CD}_{14}{ }^{+}$cells from peripheral blood and especially synovial fluid from RA patients. The expression of TREM-1 in monocytes was increased by co-culture with RASF. TLR-ligand-activated RASF further elevated TREM-1 level. Transwell assay indicated that soluble factors played a key role in RASF-promoted expression of TREM-1 in monocytes. RASF, with or without stimulation by TLR ligands, increased secretion of PGE 2 in a cyclooxygenase (COX)-2-dependent manner. $\mathrm{PGE}_{2}$ enhanced the increase in TREM-1 level in monocytes. Finally, studies using COX-2 inhibitors, COX-2 siRNA (siCOX-2) and EP1-4 antagonists, showed that RASF promotion of TREM-1 expression in monocytes was mediated by $C O X-2 / \mathrm{PGE}_{2} / \mathrm{EP}_{2,4}$ signaling.

Conclusions: Our data is the first report to reveal the critical role of RASF in upregulating TREM-1 expression in monocytes, which indicates that TREM-1 might be a novel target for RA therapy.

Keywords: Triggering receptor expressed on myeloid cells-1(TREM-1), Monocytes, Rheumatoid arthritis synovial fibroblasts (RASF), Prostaglandin E2 (PGE $)$

\section{Introduction}

Rheumatoid arthritis (RA) is a chronic inflammatory disease characterized by synovial inflammation and progressive destruction of the joints [1]. Infiltrating immune cells such as neutrophils, monocytes/macrophages, and $\mathrm{T}$ cells, proliferating rheumatoid arthritis synovial fibroblasts (RASF), extracellular matrix components, and

\footnotetext{
*Correspondence: anpingpeng9508@163.com; qubochen326@126.com ${ }^{1}$ Biological Resource Center, The Second Affiliated Hospital of Guangzhou University of Chinese Medicine, Guangzhou, Guangdong, China
} Full list of author information is available at the end of the article various inflammatory cytokines constitute the local synovial microenvironment $[2,3]$. These immune cells play a critical role in disease pathogenesis by producing various pro-inflammatory cytokines such as TNF-a, IL-1 $\beta$, and IL-6 [4], which in turn contribute to joint destruction. RASF, the dominant resident cells in the synovial microenvironment, perpetuate joint inflammation and contribute to the erosion of the cartilage and bone [5]. RASF secrete a series of inflammatory mediators: cytokines such as TNF-a, IL-6, and IL-8; chemokines such as IP-10, MCP-1, and MIP-1a; matrix metalloproteinases;

(C) The Author(s). 2019 Open Access This article is distributed under the terms of the Creative Commons Attribution 4.0 International License (http://creativecommons.org/licenses/by/4.0/), which permits unrestricted use, distribution, and 
and other pro-inflammatory molecules such as prostaglandins and leukotrienes $[4,6,7]$. Moreover, RASF are the main source of cyclooxygenase (COX)-2 in synovial tissue and product prostaglandin $\mathrm{E}_{2}\left(\mathrm{PGE}_{2}\right)$ which mediates pain and inflammatory responses in $\mathrm{RA}$. $\mathrm{PGE}_{2}$ exerts its function by interacting with PGE2 receptors, EP1 through EP4 [8]. RASF also express different toll-like receptors (TLR) such as TLR2, TLR3, and TLR4, and ligation of these receptors increases the production of inflammatory cytokines $[9,10]$. In addition, RASF can interact with infiltrating immune cells through cell-cell contact as well as elaboration of soluble factors like IL-6, IL-8, and IL-15, which promote the recruitment, activation, expansion, and production of inflammatory cytokines of infiltrating immune cells. These processes develop and maintain the inflammatory response network in the synovial microenvironment and exacerbate RA inflammation.

Triggering receptor expressed on myeloid cells-1 (TREM-1, CD354), a recently identified immunoglobulin superfamily member, mainly expresses on myeloid cells, such as neutrophils, monocytes, and tissue macrophages $[11,12]$. TREM-1 is present in two forms, a membranebound receptor form and a soluble protein form that arises from the proteolytic cleavage of the membranebound form [13]. The precise ligand for TREM-1 is not yet known. Engagement of TREM-1 on the cell surface leads to activation of a cascade of intracellular events which include the phosphorylation of phospholipase and extracellular signal-related kinase $1 / 2$ and increase nuclear levels of the nuclear factor kappa B transcription factor, and that results in the production of many chemokines such as MCP-1 and IL- 8 and inflammatory cytokines TNF-a, IL-1 $\beta$, and IL-12, degranulation of neutrophils, phagocytosis $[11,12]$, polarization of macrophages [14], activation of synovial dendritic cells [15], and negative regulation of osteoclastogenesis [16]. These responses can synergize with innate immune stimuli to amplify inflammatory responses. The soluble form of TREM-1 is released into human bloods in certain diseases, such as sepsis [17], RA [18], pneumonia [19], and primary antiphospholipid [20], and can thus serve as a biomarker for these diseases.

TREM-1 is involved in regulating infectious and noninfectious inflammation, autoimmune response, and tumor $[21,22]$. During acute inflammation, TREM-1 expression is induced by various TLR ligands [23, 24]. However, in chronic inflammation like RA, the regulatory mechanism of TREM-1 expression has not yet been elucidated. Previous studies showed that TREM-1 expression is increased in RA patients. Furthermore, $\mathrm{CD}_{14}{ }^{+}$cells are the major TREM-1-expressing cell type in RA synovium sections and synovial fluid [25, 26]. Hypoxia, an important characteristic condition of the inflamed joint, upregulates TREM-1 expression, which may promote macrophage polarization to a M1 proinflammatory state [14] and trigger dendritic cell proinflammatory cytokine and chemokine production [27]. In addition, functionally activating TREM- 1 contributes to the development or maintenance of inflammation in RA [25, 26], while inhibiting TREM-1 decreases inflammation $[28,29]$, suggesting that TREM-1 could be a new therapeutic target for RA. However, the exact mechanism of regulating TREM-1 expression remains to be defined, especially in the synovial microenvironment.

Given that monocytes and RASF are infiltrating immune cells and resident cells respectively and that they present together in the synovial microenvironment, we hypothesized that TREM-1 expression in synovial monocytes might be promoted by interaction with RASF. In this study, we investigated the expression of TREM-1 in synovial $\mathrm{CD} 14^{+}$cells to define the mechanism by which RASF regulate TREM-1 expression through a co-culture system of RASF with monocytes in vitro.

\section{Methods \\ Subjects and ethics statement}

Synovial tissue specimens used for culturing RASF $(n=5)$, osteoarthritis synovial fibroblasts (OASF; $n=5$ ), and posttraumatic synovial fibroblasts (PTSF; $n=5$ ) were obtained from patients during synovectomy or joint replacement in the Second Affiliated Hospital of Guangzhou University of Traditional Chinese Medicine and the Third Affiliated Hospital of Sun Yat-sen University. All patients fulfilled the American College of Rheumatology 2009 criteria for RA and the 1995 criteria for OA. The average DAS28 of RA patients recruited for RASF collection was 4.90, and methotrexate and glucocorticoid therapy were used before surgery. Peripheral blood used for isolating peripheral blood mononuclear cells (PBMC) and paired synovial fluids used for separating synovial cells were obtained from RA patients $(n=10)$ in the Second Affiliated Hospital of Guangzhou University of Traditional Chinese Medicine. The study was approved by the Ethics Committee of the Second Affiliated Hospital of Guangzhou University of Chinese Medicine. All procedures and methods were performed in accordance with relevant guidelines and regulations. In addition, all participants signed a written informed consent to donate sample for research.

\section{Synovial fibroblasts isolation, culture, and stimulation}

Synovial tissue samples were minced mechanically, washed with phosphate-buffered saline and seeded into tissue culture flasks for $2 \mathrm{~h}$ at $37^{\circ} \mathrm{C}$ until the fragments attached to the plates. Then, Dulbecco's modified Eagle medium (HyClone, Logan, UT) supplemented with 10\% heat-inactivated fetal bovine serum (Gibco, Carlsbad, $\mathrm{CA}), 100 \mathrm{U} / \mathrm{mL}$ penicillin, and $100 \mu \mathrm{g} / \mathrm{mL}$ streptomycin 
(Invitrogen, Carlsbad, CA) was added to the tissue culture flasks and the cells cultured in a humidified incubator containing $5 \% \mathrm{CO}_{2}$ at $37^{\circ} \mathrm{C}$. After overnight culture, nonadherent cells were removed and adherent cells were cultured. Cells from passages 4 to 7 were used. The purity of synovial fibroblast cells was more than $95 \%$ according to CD90 expression assessed using flow cytometry. For TLR ligand stimulation, RASF $\left(5 \times 10^{4} /\right.$ well $)$ in 6 -well plates were incubated with different TLR ligands for $24 \mathrm{~h}$, respectively, as follows: TLR2 ligand peptidoglycan (PGN; $10 \mu \mathrm{g} / \mathrm{mL}$ ), TLR3 ligand polyinosinic:polycytidylic acid (ployI:C; $25 \mu \mathrm{g} / \mathrm{mL}$ ), TLR4 ligand lipopolysaccharide (LPS; $100 \mathrm{ng} / \mathrm{mL}$ ) from Sigma (St. Louis, Missouri), and TLR9 ligand Type B CpG $(10 \mu \mathrm{g} / \mathrm{mL})$ from Invitrogen (Carlsbad, CA, USA). The cells were harvested for the next experiment.

\section{Preparation of $\mathrm{CD} 14^{+}$monocytes}

PBMC were separated from peripheral blood by centrifugation over Ficoll-Hypaque density gradient (Lymphoprep ${ }^{\mathrm{Tm}}$, Fresenius Kabi Norge AS, Oslo, Norway). CD14 ${ }^{+}$cells were immunomagnetically separated by positive selection from PBMC using $\mathrm{CD}_{14}{ }^{+}$monocyte isolation kit (Miltenyi Biotec, Bergisch Gladbach, Germany) according to the manufacturer's instructions, and purity was analyzed by flow cytometry (BD FACSCanto II; BD Bioscience, San Jose, CA) (purity of $\mathrm{CD} 14^{+}$cells $>95 \%$ ). $\mathrm{CD}_{1} 4^{+}$cells were pretreated with or without EP1, EP2, EP3, and EP4 antagonists (respectively, GW848687, AH6809, L798106, and AH23848; Cayman Chemicals, Ann Arbor, MI) for $2 \mathrm{~h}$ and used for the next experiment.

\section{RASF/monocytes co-culture assay}

RASF $\left(1 \times 10^{4} /\right.$ well $)$ were plated into 6 -well plates with or without $25 \mu \mathrm{g} / \mathrm{mL}$ poly(I:C) or $100 \mathrm{ng} / \mathrm{mL}$ LPS for 24 $h$. The cells were then washed with serum-free RPMI 1640 medium and co-cultured with allogeneic CD14 ${ }^{+}$ normal monocytes at a ratio of 1:100, with or without a $0.4-\mu \mathrm{m}$-pore-size membrane, for $24 \mathrm{~h}$. Only nonadherent cells (monocytes) were subsequently harvested for flow cytometry analysis.

\section{Transwell assay}

In the transwell culture system (Corning Inc., NY), passages 4-6 RASF $\left(1 \times 10^{4}\right.$ cells/well $)$ were seeded in the lower chamber and incubated overnight. Purified monocytes were added to the lower or upper chamber at a monocyte to RASF ratio of 100:1. After $24 \mathrm{~h}$, the monocytes were harvested and measured for TREM-1 expression using flow cytometry (FACSCanto, BD Biosciences), and the results were analyzed with FlowJo flow cytometry analysis software version 7.6 (Tree Star, Ashland, OR).

\section{Quantitative real-time PCR}

Total RNA was extracted from cells using TRIzol reagent (Invitrogen). Reverse transcription was performed with the RevertAid First Strand cDNA synthesis kit (Fermentas, Glen Burnie, MD) according to the manufacturer's instruction. The cDNA was subjected to quantitative real-time PCR (qPCR) analysis using SYBR Green Master Mix (Applied Biosystems, Foster City, CA) according to the manufacturer's protocol. The primers used for amplification of TLR2, TLR3, TLR4, TLR7, TLR8, TLR9, cyclooxygenase-2 (COX-2), and GAPDH were as follows: TLR2 sense primer: 5'-TCACTCAGGAGCAGCAAGCA-3', antisense primer: 5'-TGTGACATTCCGACACCGAGA-3'; TLR3 sense primer: 5'-GATCTGTCTCATAATGGCTTGT-3', antisense primer: 5'-GGCAAAGATATCCAGTTCTTCA-3'; TLR4 sense primer: 5'-AGCCTAAGCCACCTCTCTACCT-3', antisense primer: 5'-AGATTTGTCTCCACAGCCACCA3'; TLR7 sense primer: 5'-AGCTTTAACCTCTCGCCATTACA-3', antisense primer: 5'-TTGAGCAGAAGCCAACTTCACT-3'; TLR8 sense primer: 5'-CTTC AGTCGTCAATGCTGACCT-3', antisense primer: 5' GATTGCTGCACTCTGCAATAACT-3'; TLR9 sense primer: 5'-CCGTGGCAATGTCACCAG-3', antisense primer 5'-GCAGTTCCACTTGAGGTTGAG-3'; COX-2 sense primer: 5'-CCAGCACTTCACGCATCAGT-3', antisense primer: 5'-TGTCTAGCCAGAGTTTCACCGT-3'; GAP DH sense primer: $5^{\prime}$-TGTTCGTCATGGGTGTGAACCA3', antisense primer: 5'-GTCATGAGTCCTTCCACGATACCA-3'. Gene expression was quantified relative to the expression of the housekeeping gene GAPDH and normalized to the control by the standard $2^{-\Delta \Delta C t}$ calculation.

\section{RNA interference}

Following the manufacturer's instructions for transfection, RASF was seeded on 6-well plates and cultured until 80\% confluent. Then, siRNA oligonucleotides targeting human COX-2 mRNA (siCOX-2) and a non-related control siRNA (Santa Cruz Biotechnology, Santa Cruz) were transfected into the RASF using Lipofectamine RNAiMAX transfection reagent (Invitrogen) for $6 \mathrm{~h}$. Cells were washed and added into the DMEM medium with $10 \%$ FBS to be cultivated for $48 \mathrm{~h}$. After the cells were harvested, COX-2 protein expression was measured by Western blot; the procedure yielded an average of $80 \%$ COX-2 expression reduction. After transfection, RASF were used for co-culture experiments.

\section{Flow cytometry}

Collected cells were stained with FITC-conjugated human anti-CD14 and APC-conjugated human anti-TREM-1 (Biolegend, San Diego, CA). TREM-1 protein levels on the CD14-gated cells were measured using flow cytometry (FACSCanto) and analyzed with FlowJo software version 
7.6 (Tree Star). Isotype-matched monoclonal antibodies (Biolegend) were used as negative controls.

\section{ELISA}

The concentration of $\mathrm{PGE}_{2}$ in the culture supernatant was determined by using a $\mathrm{PGE}_{2}$ ELISA kit (R\&D Systems, Minneapolis, MN) according to the manufacturer's instructions.

\section{Statistical analysis}

Data are presented as means \pm SD. All statistical analysis was performed with SPSS 19.0 statistical software (IBM, Endicott, NY). Comparisons of 2 groups were analyzed using two-tailed Student's $t$ test. Comparison of 3 or more groups was analyzed using ANOVA with Bonferroni's post hoc test when multiple pairwise comparisons were made between different groups. Statistical significance for all tests was set as $P<0.05$.

\section{Results}

TREM-1 expression is higher in $\mathrm{CD}_{14^{+}}$synovial cells than $\mathrm{CD}_{14}{ }^{+}$peripheral blood monocytes from RA patients

The characteristics of the $10 \mathrm{RA}$ patients are presented in Table 1. A total of $78.6 \%$ of the patients were positive for rheumatoid factor, $84.1 \%$ of patients were positive for antiCCP antibodies, and $69.2 \%$ of patients were positive for both. All RA patients had active disease, and none of them were receiving any immunosuppressive treatment at the time of the study. RA synovial fluid is a local inflammatory microenvironment; whether TREM-1 expression in $\mathrm{CD}_{1} 4^{+}$synovial cells differs from that of $\mathrm{CD}_{1} 4^{+}$peripheral blood monocytes in RA patients remains unclear. So, we used flow cytometry analysis to study the expression of TREM-1 in $\mathrm{CD} 14^{+}$peripheral blood monocytes from RA patients and healthy donors. The results showed that TREM-1 expression was upregulated in $\mathrm{CD} 14^{+}$blood monocytes from RA patients compared with those from healthy controls, consistent

Table 1 Summary of RA patient characteristics (mean \pm SD)

\begin{tabular}{lll}
\hline & $\begin{array}{l}\text { RA } \\
(n=10)\end{array}$ & $\begin{array}{l}\text { Healthy control (HC) } \\
(n=10)\end{array}$ \\
\hline Age & $51.2 \pm 5.7$ & $45.4 \pm 8.9$ \\
Female, $n(\%)$ & $7(70)$ & $6(60)$ \\
DAS28 (ESR) & $4.5 \pm 0.5$ & NA \\
Disease duration & $9.3 \pm 3.1$ & NA \\
RF positive (\%) & 78.6 & NA \\
Anti-CCP positive (\%) & 84.1 & NA \\
Positive for both RF and anti-CCP (\%) & 69.2 & NA \\
CRP (mg/L) & $12.7 \pm 10.5$ & ND \\
ESR (mm/h) & $82.3 \pm 12.7$ & ND \\
\hline
\end{tabular}

DAS28 disease activity score $28, R F$ rheumatoid factor, anti-CCP anti-cyclic citrullinated peptide antibodies, CRP C-reactive protein, ESR erythrocyte sedimentation rate, NA not applicable, ND not determined with previous reports [25, 26] (Fig. 1a, b). Moreover, TREM1 expression was significantly higher in $\mathrm{CD}_{1} 4^{+}$synovial cells than in $\mathrm{CD} 14^{+}$blood monocytes of 10 independent RA patients (Fig. 1a, b).

\section{TREM-1 expression in monocytes is upregulated by soluble factors co-cultured with RASF}

RASF are the principal resident cells of the synovial membrane where they interact with immune cells, including monocytes/macrophages, lymphocytes, and mast cells. We next investigated whether the higher expression of TREM-1 in infiltrating monocytes of synovial fluid was specifically related to RASF. An in vitro co-culture system of human normal $\mathrm{CD}_{1}{ }^{+}$monocytes and RASF was developed with various mixing ratios and for several indicated time points, and TREM-1 expression in monocytes was detected by flow cytometry. The CD14 $4^{+}$TREM $-1^{+}$flow cytometric dot plots only show the percentage of TREM-1 positive expressing monocytes, but flow cytometric histograms of TREM-1 expression on gated CD14 monocytes show total TREM-1 expression including the enhanced percentage of TREM-1 positive expressing monocytes but also the enhanced expression of TREM-1 in single monocytes. Co-culture of monocytes with RASF led to increased TREM-1 expression by both increasing number of TREM-1 expressing monocytes and inducing the TREM-1 level in TREM-1 positive expressing cells. So we chose flow cytometric dot plots combined with histograms instead of single flow cytometric dot plots to show TREM-1 level in monocytes. Furthermore, TREM-1 expression peaked at a RASF-to-monocyte ratio of 1:100 (Fig. 2a) and co-culture for $24 \mathrm{~h}$ (Fig. 2b), so the 1:100 mixed cell ratio and 24-h co-culture were selected for subsequent experiments. In addition, monocytes that were co-cultured with PTSF or OASF did not show any increase in the expression of TREM-1 (Fig. 2c), suggesting that the increased expression of TREM-1 in monocytes of synovial fluid is regulated by RASF specifically. TREM-1 level in RASF was also detected by flow cytometry and Additional file 1: Figure S1 showed that TREM-1 was not expressed in RASF.

Next, we performed transwell experiments by separating monocytes from RASF using a permeable membrane to prevent direct contact, but allowing soluble factors to pass through. Monocytes were seeded in the upper chamber, and allogeneic RASF were seeded in the lower chamber. For comparison purposes, monocytes were also cultured alone or with allogeneic RASF in the lower chamber. There was no difference in results between addition of RASF to the chamber separated from direct contact with monocytes and addition of RASF to the monocyte co-culture (Fig. 2d). Thus, the transwell assay indicates that soluble factors, but not direct cell contact, are required for RASF to positively regulate TREM-1 expression in monocytes. 


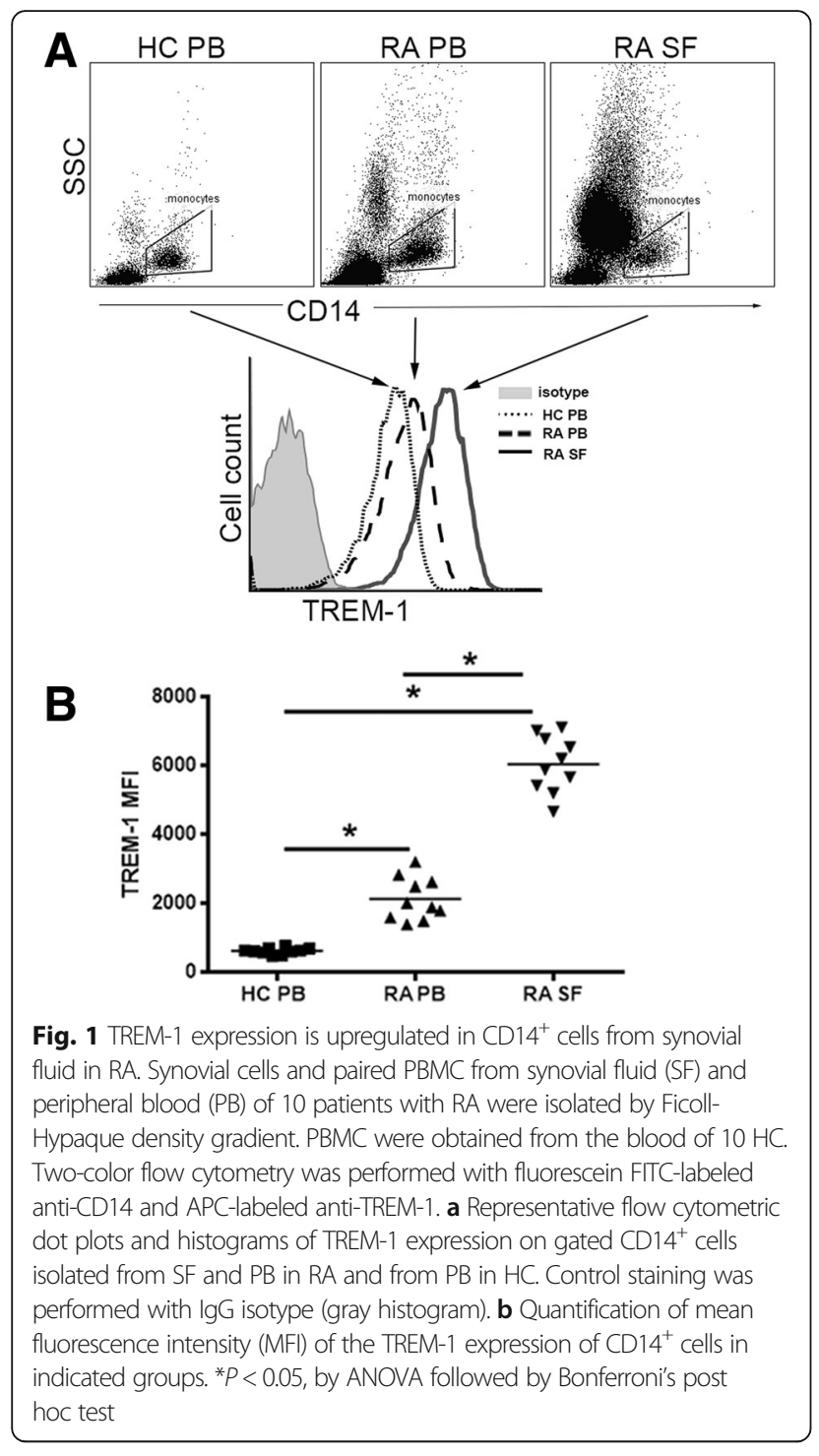

RASF enhance TREM-1 level in monocytes through the COX-2/PGE 2 /EP2,4 pathway

Elevated COX-2 expression and $\mathrm{PGE}_{2}$ secretion have been observed in RASF, and RASF are considered the main cellular source of COX-2 in RA patients [1, 3, 30]. Moreover, $\mathrm{PGE}_{2}$ promotes the expression of TREM-1 in macrophages induced by LPS [31] or human non-small-cell lung cancerassociated macrophages [32]. We therefore investigated whether high expression of TREM-1 in synovial monocytes was induced by $\mathrm{PGE}_{2}$ production from RASF by the cyclooxygenase pathway. We first confirmed that both the expression of COX-2 (Fig. 3a) and the secretion of $\mathrm{PGE}_{2}$ (Fig. 3b) were increased in RASF compared to PTSF or OASF. Next, we treated monocytes with $\mathrm{PGE}_{2}$. The level of TREM-1 was obviously increased in monocytes stimulated by exogenous $\mathrm{PGE}_{2}$ (Fig. 3c). To confirm the role of COX-2 in TREM-1 induction, RASF were pre-treated with COX-2 inhibitors or vehicle for $8 \mathrm{~h}$, then co-cultured with normal human monocytes for $24 \mathrm{~h}$. The level of TREM-1 in monocytes was determined using flow cytometry. Monocytes cocultured with RASF showed an increased expression of TREM-1, while treatment with a COX-2 inhibitor (Celecoxib, $100 \mu \mathrm{mol}$ ) resulted in a considerable decrease in TREM-1 induction (Fig. 3d). We further knocked down COX-2 expression in RASF by employing siCOX-2 and then co-cultured these pre-treated RASF with normal human monocytes for $24 \mathrm{~h}$. Flow cytometry data showed that treatment with siCOX-2 also led to a reduction in TREM-1 expression (Fig. 3e). To further identify the receptors by which $\mathrm{PGE}_{2}$ regulates the expression of TREM-1, we performed experiments with antagonists of EP1 through EP4. Monocytes were treated with the respective antagonist prior to coculturing with RASF. Antagonists of EP2 (AH6809) and EP4 (AH23848) downregulated the expression of TREM-1, but antagonists of EP1 (GW848687) and EP3 (L798106) had no effect on the expression of TREM-1 (Fig. 3f).

\section{TLR-ligand-stimulated RASF also enhance the expression of TREM-1 in monocytes through the COX-2/PGE 2 pathway}

Previous studies indicated that RASF excessively expresses TLR and that TLR stimulation can induce the production of both pro-inflammatory and anti-inflammatory cytokines $[9,10]$. Therefore, we first detected the expression of TLR in RASF by qPCR. TLR2, TLR3, TLR4, TLR7, TLR8, and TLR9 were all expressed at higher levels in RASF than in PTSF and OASF (Fig. 4a). To confirm whether TLR-ligand-activated RASF enhanced TREM-1 expression in monocytes, we stimulated RASF with different TLR ligands and co-cultured these RASF with monocytes, then observed TREM-1 levels by flow cytometry. As shown in Fig. 4b, pre-treating RASF with ligands of TLR2 (PGN), TLR3 (polyI:C), or TLR4 (LPS) resulted in higher expression of TREM-1 in monocytes, while pre-treating RASF with TLR7/8 ligand (R848) or TLR9 ligand (CpG) had no influence on TREM-1 expression in monocytes.

We next investigated whether TLR2-, TLR3-, or TLR4-enhanced TREM-1 expression also depended on the activation of the $\mathrm{COX}-2 / \mathrm{PGE}_{2}$ signaling pathway. We first confirmed using qPCR that RASF stimulated with TLR3 or TLR4 ligand expressed a high level of COX-2 (Fig. 4c) and using ELISA that they secreted a higher amount of $\mathrm{PGE}_{2}$ (Fig. 4d), but that TLR2-ligandstimulated RASF did not affect COX-2 or $\mathrm{PGE}_{2}$ (Fig. 4c, d). In addition, both TLR7/8 ligand and TLR9 ligand had no effect on COX-2 mRNA expression and $\mathrm{PGE}_{2}$ secretion in RASF (Additional file 2: Figure S2). Furthermore, RASF were transfected with siCOX-2 and then stimulated with poly I:C or LPS respectively, then cocultured with monocytes for $24 \mathrm{~h}$ to confirm the role of COX-2 induction of TREM-1. Flow cytometry data 

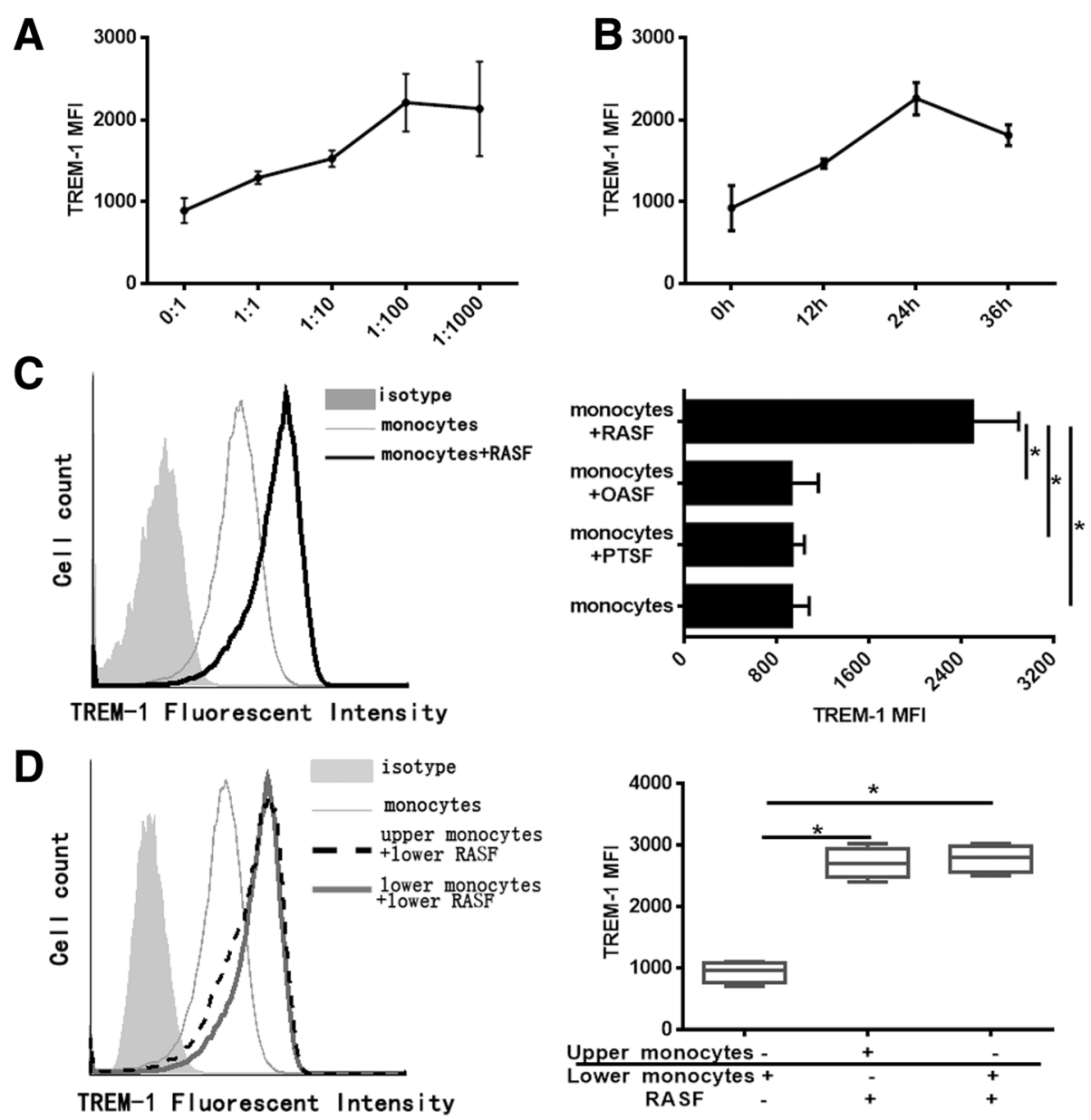

Fig. 2 TREM-1 expression in monocytes is enhanced co-cultured with RASF. a Monocytes were co-cultured with RASF $(n=5)$ with various mixing ratios for $24 \mathrm{~h}$. TREM-1 expression on the CD14-gated cells was assessed using flow cytometry. b Monocytes were co-cultured with RASF $(n=5)$ with 100:1 ratio for various cultural time. TREM-1 level on the CD14-gated cells were detected by flow cytometry. c After monocytes were cocultured with RASF $(n=5)$ or OASF $(n=5)$ or PTSF $(n=5)$ with 100:1 ratio for $24 \mathrm{~h}$, TREM-1 expression in monocytes was assessed using flow cytometry. The left panel was the representative flow cytometric histograms, and the right panel was a statistical chart. d Transwell assay was performed in which monocytes were separated from allogeneic RASF; as comparison, monocytes were cultured together with RASF ( $n=5)$. TREM-1 expression on the CD14-gated cells was shown as the mean fluorescence intensity (MFI) values. The left panel was the representative flow cytometric histograms, and the right panel was a statistical chart. ${ }^{*} P<0.05$, by ANOVA followed by Bonferroni's post hoc test

showed that there was a considerable reduction of TREM-1 expression in monocytes (Fig. 4e, f).

\section{Discussion}

Our results showed that TREM-1 expression was significantly enhanced in $\mathrm{CD} 14^{+}$synovial cells of RA patients, compared with $\mathrm{CD} 14^{+}$peripheral blood monocytes and healthy controls. Furthermore, only RASF, and not OASF or PTSF, showed the effect of upregulation of TREM-1 in monocytes, indicating that the role of RASF in TREM-1 expression is specific for RA. It was noting that RASF not only increased number of TREM-1 expressing monocytes, but also induced the TREM-1 level in TREM-1 positive expressing cells. TLR-ligandactivated RASF further promoted the increased TREM-1 level. Additionally, RASF with or without TLR ligand stimulation showed increased secretion of $\mathrm{PGE}_{2}$ in a COX-2-dependent manner, and monocytes treated with $\mathrm{PGE}_{2}$ showed significantly increased TREM-1 level. Finally, both treatment with COX-2 inhibitor and knockdown of COX-2 in RASF attenuated the expression of TREM-1 in monocytes in a co-culture model of RASF with monocytes. Taken together, these data suggest that TREM-1 might be a critical link between infiltrating $\mathrm{CD}_{14}{ }^{+}$cell activation and chronic inflammatory response in the synovial microenvironment.

The excessive and persistent activation of the immune system is a central factor for chronic synovial inflammation, which is intricately at play in both resident and infiltrating cells [33-36]. A massive influx of activated monocytes has been demonstrated in the inflamed joints of RA patients [33]. Monocytes, as essential innate 
A

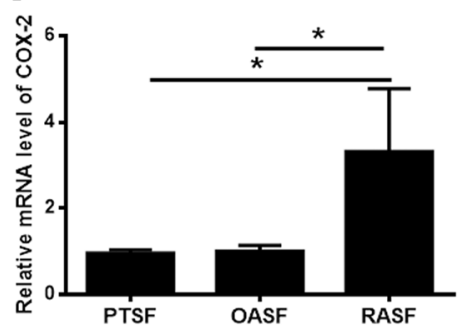

C

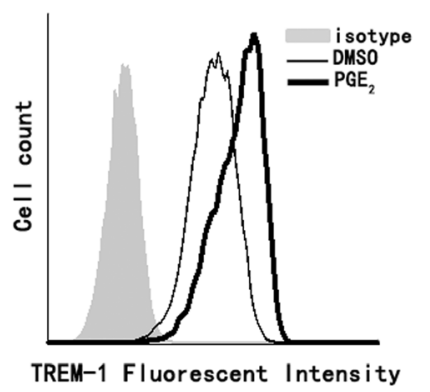

D

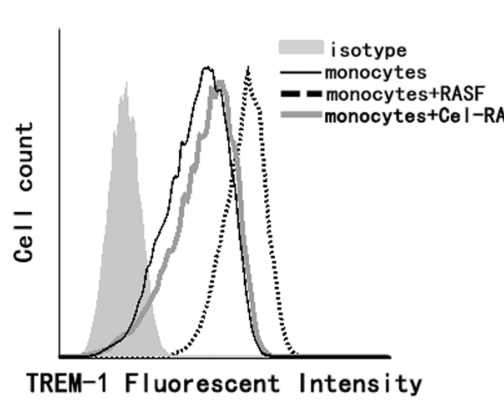

B
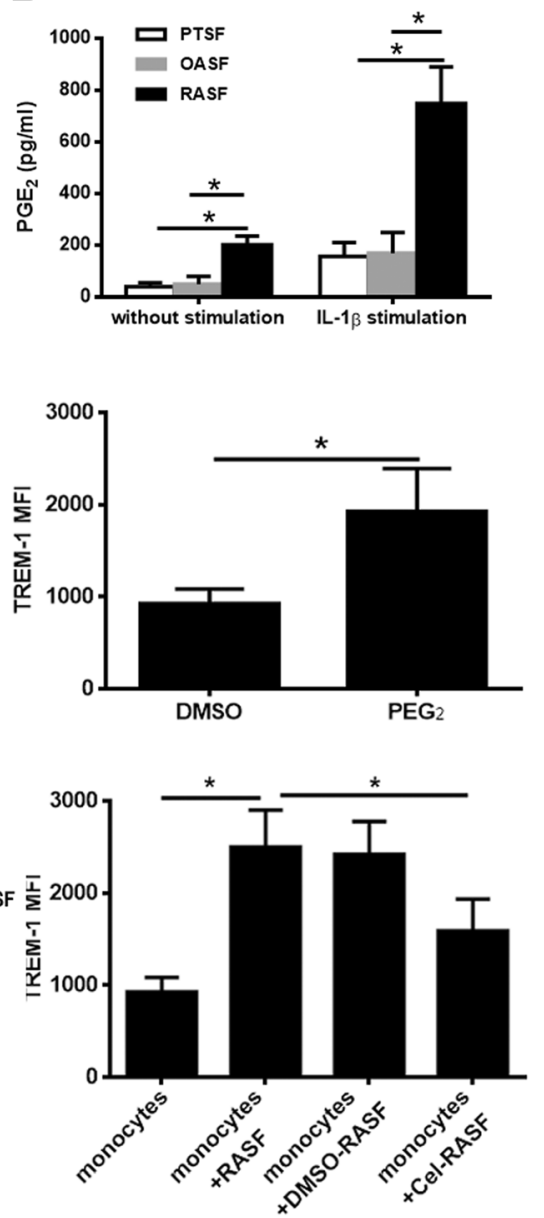
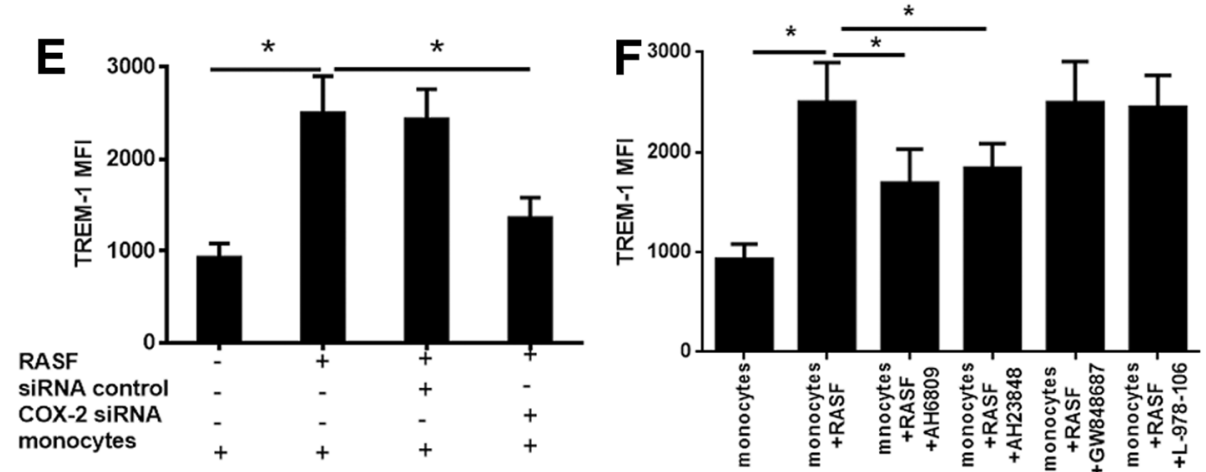

Fig. 3 RASF promote TREM-1 level in monocytes through the COX-2/PGE 2 pathway. a Total RNA was reverse-transcribed, and the mRNA level of COX- 2 in RASF $(n=5)$ or OASF $(n=5)$ or HCSF $(n=5)$ was detected by qPCR. b After RASF or OASF or PTSF were cultured for $24 \mathrm{~h}$ with or without 10 $\mathrm{ng} / \mathrm{mL} \mathrm{IL}-1 \beta$ stimulation, the cell culture supernatants were collected for PGE $\mathrm{E}_{2}$ ELISA. c After stimulation with recombinant PGE 2 or vehicle, TREM-1 expression in $\mathrm{CD}_{1} 4^{+}$monocytes was measured by flow cytometry. The left panel was the representative flow cytometric histograms, and the right panel was a statistical chart. $\mathbf{d}$ After CD14 $4^{+}$monocytes were co-cultured with RASF $(n=5)$ pre-treated with or without of Celecoxib (Cel, $\left.100 \mu \mathrm{mol}\right)$ (specific COX-2 inhibitor) for $24 \mathrm{~h}$, TREM-1 level in CD14 $4^{+}$monocytes were measured using flow cytometry. The left panel was the representative flow cytometric histograms, and the right panel was a statistical chart. e After CD14 $4^{+}$monocytes co-cultured with RASF $(n=5)$ expressing control siRNA or COX-2 siRNA for $24 \mathrm{~h}$, the expression of TREM-1 in monocytes was detected by flow cytometry. $\mathbf{f}$ After CD14 $4^{+}$monocytes pre-treated with antagonists of EP1-4 (GW848687, AH6809, L798106, and AH23848) were co-cultured with RASF $(n=5)$ for $24 \mathrm{~h}$, TREM-1 expression in monocytes was detected by flow cytometry. ${ }^{*} P<0.05$, by ANOVA followed by Bonferroni's post hoc test $(\mathbf{a}, \mathbf{b}, \mathbf{d}, \mathbf{e}$, and $\mathbf{f})$ and two-tailed Student's $t$ test (c) 

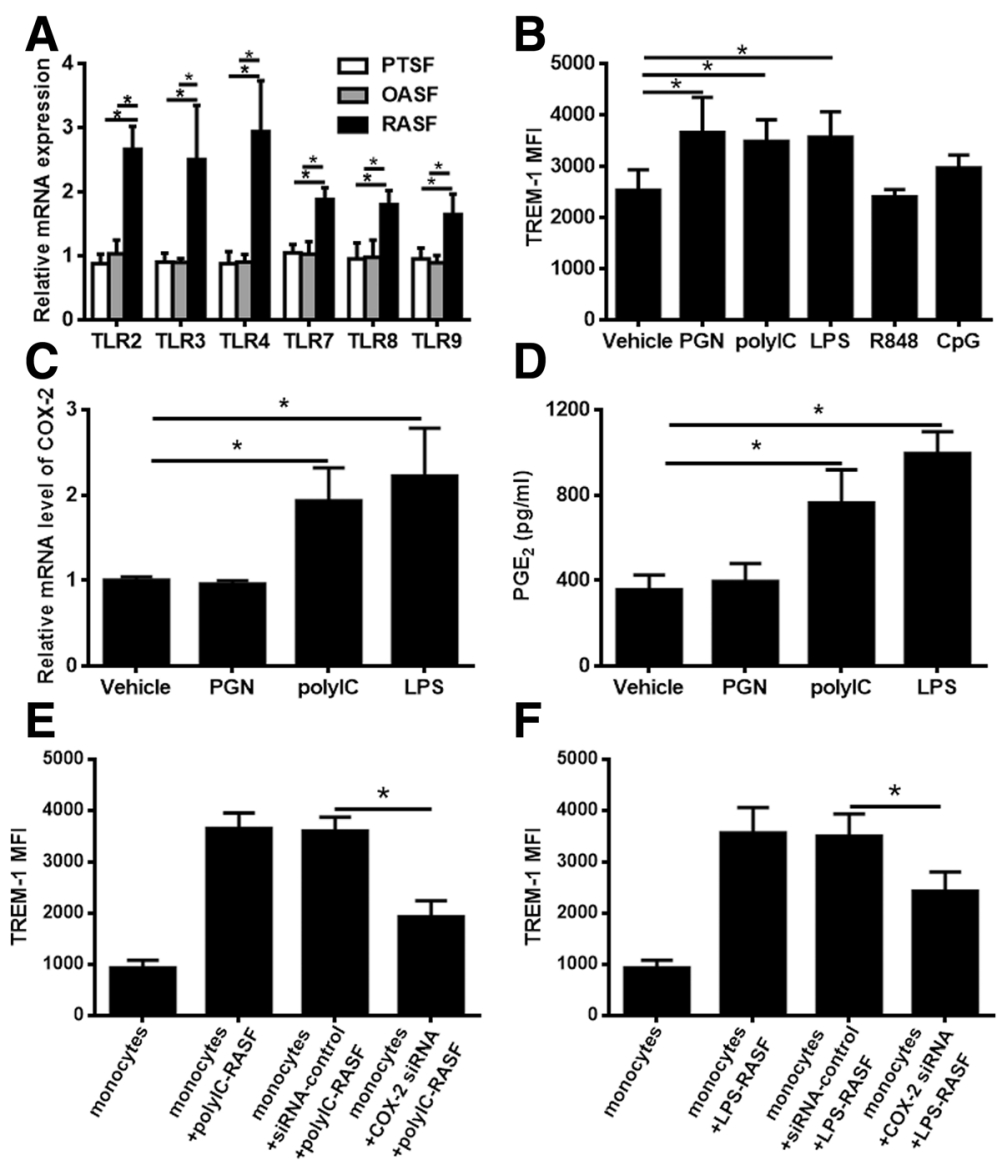

F

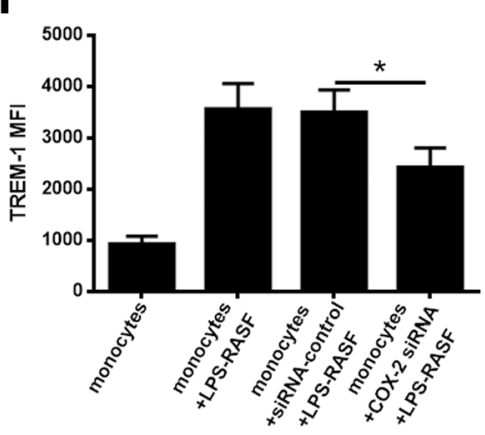

Fig. 4 Activated RASF promote TREM-1 expression in monocytes through the COX-2/PGE 2 pathway. a TLR2, TLR3, TLR4, TLR7, TLR8, and TLR9 levels in RASF ( $n=5$ ) were detected by qPCR. b After stimulation with $1 \mu \mathrm{g} / \mathrm{mL} \mathrm{LPS}, 10 \mu \mathrm{g} / \mathrm{mL}$ PGN, $25 \mu \mathrm{g} / \mathrm{mL}$ poly(l:C), $10 \mu \mathrm{g} / \mathrm{mL}$ CpG respectively, RASF $(n=5)$ were co-cultured with allogeneic normal monocytes for $24 \mathrm{~h}$. The monocytes were harvested for flow cytometry analysis of the TREM-1 expression. After stimulation with DMSO, PGN, poly I:C and LPS respectively, $\mathbf{c}$ the mRNA level of COX-2 in RASF $(n=5)$ was detected by qPCR, and $\mathbf{d}$ the secretion of $\mathrm{PGE}_{2}$ in RASF $(n=5)$ was measured using ELISA. RASF expressing control siRNA or COX-2 siRNA were stimulated with e poly l:C or $\mathbf{f} L P S$ and then cocultured with $\mathrm{CD} 14^{+}$monocytes for $24 \mathrm{~h}$. TREM-1 expression in CD14+ monocytes was detected by flow cytometry. ${ }^{*} P<0.05$, by ANOVA followed by Bonferroni's post hoc test ( $\mathbf{a}, \mathbf{e}$, and $\mathbf{f}$ ) and two-tailed Student's $t$ test $(\mathbf{b}, \mathbf{c}$, and $\mathbf{d})$

immune cells, can produce a wide range of proinflammatory cytokines, chemokines, and other factors; polarize $\mathrm{CD}^{+}{ }^{+} \mathrm{T}$ cells $[33,37]$; and contribute to RASF activation, proliferation, migration, and invasion [3840]. RASF, as key resident cells, also contribute actively to inflammation [5]. More and more evidence reveals that RASF can inhibit the monocyte apoptotic process [41] and induce their activation $[5,40]$. In turn the increased inflammatory monocytes induce more robust RASF activation and proliferation [38], forming a positive feedback loop that enhances and perpetuates the inflammatory response. Previous studies have found that TREM-1 expression and activation may elicit and contribute to monocyte activation and inflammatory cytokine secretion [11, 42-45]. A novel finding in our study is that RASF significantly increase TREM-1 expression in synovial monocytes. Therefore, our data support the hypothesis that RASF increase the inflammatory response in monocytes by regulating TREM-1 expression, and then inflammatory monocytes consequently induce more robust RASF activation. This forms a costimulatory circuit that enhances the inflammatory microenvironment, recruits and activates more immune cells, and ultimately contributes to the chronic inflammatory response. Taken together, these findings suggest that TREM-1 may be a potential immunotherapeutic target in RA.

We next studied the mechanism of how RASF promote the expression of TREM-1 in synovial monocytes. Transwell assay indicated that the upregulation of TREM-1 level by RASF is independent of direct cell contact but is dependent only on soluble factors. Since previous studies indicated that TREM-1 expression is modulated by lipid mediators, particularly prostaglandin $[31,32,46]$, we investigated the role of the cyclooxygenase pathway on RASF-induced TREM-1 expression. 
Prostaglandin synthetase, or COX, has at least two isoforms: COX-1, which has constitutive expression, and COX-2, which has inducible expression. COX-2, but not COX-1, is relevant to $\mathrm{PGE}_{2}$ biosynthesis in RA joints. Selective inhibition of COX-2 and neutralization of $\mathrm{PGE}_{2}$ can both ameliorate RA inflammation $[6,47,48]$. We find that both the expression of COX-2 and the secretion of $\mathrm{PGE}_{2}$ are increased in RASF, which is consistent with previous studies $[6,47,48]$. RASF-derived $\mathrm{PGE}_{2}$ obviously increases the expression of TREM-1 in monocytes. Additionally, pre-treatment of RASF with a COX2 inhibitor or siCOX-2 results in attenuation of TREM-1 expression. These findings suggest that RASF promotion of TREM-1 expression in synovial monocytes is mediated by the COX-2-PGE ${ }_{2}$ signaling. $\mathrm{PGE}_{2}$ exerts its function by interacting with PGE2 receptors, EP1 through EP4 [8]. Therefore, we treated monocytes with antagonists to EP1-4. Only the EP2 and EP4 antagonists inhibited TREM-1 expression. Thus, our study demonstrates that the expression of TREM-1 in synovial monocytes is regulated by the $\mathrm{COX}-2 / \mathrm{PGE}_{2} / \mathrm{EP} 2,4$ signaling pathway of RASF. However, inhibition of COX-2 fails to completely suppress RASF-induced TREM-1 expression in monocytes, which also suggests that the endogenous $\mathrm{PGE}_{2}$-mediated pathway seems to be one of the mechanisms and other unknown factors and pathways might also participate in the upregulation of TREM-1 expression in synovial monocytes. Other researchers report that besides $\mathrm{PGE}_{2}$, soluble factors such as TGF- $\beta$ [49], TNF- $\alpha[50,51]$, and monosodium urate monohydrate [52] can also induce TREM-1 expression, and most of these are also produced by RASF. Therefore, more indepth studies are needed to elucidate the mechanism by which RASF regulate TREM-1 in synovial monocytes.

"Pathogen-associated molecular patterns (PAMP)," such as viral and bacterial DNA and peptidoglycan, or "dangerassociated molecular patterns (DAMP)" such as high mobility group box1 protein, double-stranded RNA and heat shock proteins, have been demonstrated in inflamed RA joints $[53,54]$. This implies that PAMP or DAMP activation of TLR plays a role in RA pathogenesis. In this study, we proved that TLR activation further enhances RASFmediated upregulation of TREM-1 expression in monocytes. This synergistic effect implies that activated RASF could express high levels of COX-2 and secrete more PGE $_{2}$ after TLR3 and TLR4 ligation. This suggests that during infection or endogenous danger molecule exists, TLR serve as important contributors to RASF-mediated regulation of TREM-1 expression in monocytes, which eventually exacerbates RA inflammation. But TLR2-enhanced TREM-1 expression was not dependent on the activation of the COX-2/PGE 2 signaling pathway. TLR2-enhanced TREM-1 expression was dependent on MyD88 expression and activation of NF-kB signaling pathway $[55,56]$.
Several limitations of our study should be noted here. We focused on the effects of synovial fibroblasts on the expression of TREM-1 by monocytes in the synovial microenvironment. The varying expression of TREM-1 may be not only due to differences in the synovial tissue microenvironment and pathogenic factors in the RA synovium, but also due to differences in lineage, such as tissue-resident macrophages. Whether lineage differences share a common mechanism causing upregulation of TREM-1 expression in CD14 ${ }^{+}$synovial cells still needs to be evaluated. Moreover, we did not evaluate how the enhanced expression of TREM-1 in CD14 ${ }^{+}$synovial cells promotes synovial inflammation and RA disease progression. Further studies will define the role of TREM-1 in synovial immunomodulation in the RA synovial microenvironment.

\section{Conclusions}

In summary, our study reveals a new relationship between RASF and monocytes by modulating TREM-1 expression in the local synovial microenvironment. TREM1 expression in synovial monocytes is dependent on the cyclooxygenase pathway and is mediated by increased secretion of $\mathrm{PGE}_{2}$ by RASF. Targeting TREM-1 may potentially have therapeutic benefits in RA.

\section{Additional files}

Additional file 1: Figure S1. TREM-1 was not expressed in RASF. The TREM-1 level in RASF $(n=3)$ was detected by flow cytometry. a Left panel was the representative flow cytometric histograms and $b$ right panel was statistical chart. (TIF $271 \mathrm{~kb}$ )

Additional file 2: Figure S2. Both R848 and CpG had no effect on COX-2 mRNA expression and PGE2 secretion in RASF. a The mRNA level of COX-2 in RASF ( $n=3$ ) was detected by qPCR, and b the secretion of $\mathrm{PGE}_{2}$ in $\operatorname{RASF}(n=3)$ was measured using ELISA. (TIF $137 \mathrm{~kb}$ )

\section{Abbreviations}

Anti-CCP: Anti-cyclic citrullinated peptide antibodies; COX: Cyclooxygenase; CRP: C-reactive protein; DAMP: Danger-associated molecular patterns; DAS28: Disease activity score 28; ESR: Erythrocyte sedimentation rate; HC: Healthy controls; OASF: Osteoarthritis synovial fibroblasts;

PAMP: Pathogen-associated molecular patterns; PBMC: Peripheral blood mononuclear cells; PGE 2 : Prostaglandin E2; PTSF: Post-traumatic synovial fibroblasts; RA: Rheumatoid arthritis; RASF: Rheumatoid arthritis synovial fibroblasts; RF: Rheumatoid factor; TLR: Toll-like receptors; TREM-1: Triggering receptor expressed on myeloid cells-1

\section{Acknowledgements}

This work was supported by a grant obtained from the Natural Science Foundation of China (81303287, 81503317), the Guangdong Provincial Project of Science and Technology (2016A020215133), and the Hospital Project of Science and Technology (YN2015MS12).

\section{Authors' contributions}

AP designed the research and drafted the manuscript. $A P, X L, J H$, and $M H$ performed the experiments. $A P, X L, J H, J X$, and $H H$ analyzed the data. QC supervised the entire project. All authors reviewed the manuscript. All authors read and approved the final manuscript. 


\section{Funding}

This work was supported by a grant obtained from the Natural Science Foundation of China (81303287, 81503317), the Guangdong Provincial Project of Science and Technology (2016A020215133), and the Hospital Project of Science and Technology (YN2015MS12).

\section{Availability of data and materials}

All data supporting our findings are contained within the manuscript

\section{Ethics approval and consent to participate}

This study protocol was reviewed and approved by the Institutional Review Board at the Second Affiliated Hospital of Guangzhou University of Chinese Medicine, and all participants provided written informed consent.

\section{Consent for publication}

Not applicable.

\section{Competing interests}

The authors declare that they have no competing interests.

\section{Author details}

${ }^{1}$ Biological Resource Center, The Second Affiliated Hospital of Guangzhou University of Chinese Medicine, Guangzhou, Guangdong, China. 2Department of Pathogenic Biology and Immunology, Guangzhou Medical University, Guangzhou, China. ${ }^{3}$ Department of Laboratory Science, The Second Affiliated Hospital of Guangzhou University of Chinese Medicine, Guangzhou, China.

\section{Received: 18 February 2019 Accepted: 28 June 2019}

\section{Published online: 08 July 2019}

\section{References}

1. Muller-Ladner U, Ospelt C, Gay S, Distler O, Pap T. Cells of the synovium in rheumatoid arthritis. Synovial fibroblasts. Arthritis Res Ther. 2007;9:223.

2. Katschke KJ, Rottman JB, Ruth JH, Qin S, Wu L, LaRosa G, Ponath P, Park CC, Pope RM, Koch AE. Differential expression of chemokine receptors on peripheral blood, synovial fluid, and synovial tissue monocytes/ macrophages in rheumatoid arthritis. Arthritis Rheum. 2001:44:1022-32.

3. Nissinen $R$, Leirisalo-Repo $M$, Tiittanen $M$, Julkunen $H$, Hirvonen $H$, Palosuo $T$, Vaarala O. CCR3, CCR5, interleukin 4, and interferon-gamma expression on synovial and peripheral T cells and monocytes in patients with rheumatoid arthritis. J Rheumatol. 2003;30:1928-34.

4. Brennan FM, Mclnnes IB. Evidence that cytokines play a role in rheumatoid arthritis. J Clin Invest. 2008;118:3537-45.

5. Neumann E, Lefevre S, Zimmermann B, Gay S, Muller-Ladner U. Rheumatoid arthritis progression mediated by activated synovial fibroblasts. Trends Mol Med. 2010:16:458-68.

6. Kontny E, Rudnicka W, Kowalczewski J, Marcinkiewicz J, Maslinski W. Selective inhibition of cyclooxygenase 2-generated prostaglandin E2 synthesis in rheumatoid arthritis synoviocytes by taurine chloramine. Arthritis Rheum. 2003;48:1551-5.

7. Falconer J, Murphy AN, Young SP, Clark AR, Tiziani S, Guma M, Buckley CD. Review: synovial cell metabolism and chronic inflammation in rheumatoid arthritis. Arthritis Rheumatol. 2018:70:984-99.

8. Kalinski P. Regulation of immune responses by prostaglandin E2. J Immunol. 2012;188:21-8

9. Ultaigh SN, Saber TP, McCormick J, Connolly M, Dellacasagrande J, Keogh B, McCormack W, Reilly M, O'Neill LA, McGuirk P, et al. Blockade of Toll-like receptor 2 prevents spontaneous cytokine release from rheumatoid arthritis ex vivo synovial explant cultures. Arthritis Res Ther. 2011;13:R33.

10. He C, Lai P, Wang J, Zhou T, Huang Z, Zhou L, Liu X. TLR2/4 deficiency prevents oxygen-induced vascular degeneration and promotes revascularization by downregulating IL-17 in the retina. Sci Rep. 2016;6: 27739.

11. Bouchon A, Dietrich J, Colonna M. Cutting edge: inflammatory responses can be triggered by TREM-1, a novel receptor expressed on neutrophils and monocytes. J Immunol. 2000;164:4991-5.

12. Bouchon A, Facchetti F, Weigand MA, Colonna M. TREM-1 amplifies inflammation and is a crucial mediator of septic shock. Nature. 2001;410: 1103-7.
13. Gibot S, Kolopp-Sarda MN, Bene MC, Cravoisy A, Levy B, Faure GC, Bollaert PE. Plasma level of a triggering receptor expressed on myeloid cells-1: its diagnostic accuracy in patients with suspected sepsis. Ann Intern Med. 2004;141:9-15.

14. Raggi F, Pelassa S, Pierobon D, Penco F, Gattorno M, Novelli F, Eva A, Varesio L, Giovarelli M, Bosco MC. Regulation of human macrophage M1-M2 polarization balance by hypoxia and the triggering receptor expressed on myeloid Cells-1. Front Immunol. 2017;8:1097.

15. Canavan M, Walsh AM, Bhargava V, Wade SM, McGarry T, Marzaioli V, Moran B, Biniecka M, Convery H, Wade S, et al. Enriched Cd141+ DCs in the joint are transcriptionally distinct, activated, and contribute to joint pathogenesis. JCl Insight. 2018;3:95228.

16. Lee B, Kim JH, Jung JH, Kim TH, Ji JD. TREM-1, a negative regulator of human osteoclastogenesis. Immunol Lett. 2016;171:50-9.

17. Ravetti CG, Moura AD, Vieira EL, Pedroso ER, Teixeira AL. sTREM-1 predicts intensive care unit and 28-day mortality in cancer patients with severe sepsis and septic shock. J Crit Care. 2015:30:440-7.

18. Choi ST, Kang EJ, Ha YJ, Song JS. Levels of plasma-soluble triggering receptor expressed on myeloid cells-1 (sTREM-1) are correlated with disease activity in rheumatoid arthritis. J Rheumatol. 2012;39:933-8.

19. Palazzo SJ, Simpson T, Schnapp LM. Triggering receptor expressed on myeloid cells type 1 as a potential therapeutic target in sepsis. Dimens Crit Care Nurs. 2012:31:1-6.

20. Edel Y, Kliminski V, Pokroy-Shapira E, Oren S, Dortort LA, Pri-Paz BY, Egbaria M, Molad Y. Elevated plasma level of soluble triggering receptor expressed on myeloid cells-1 is associated with inflammation activity and is a potential biomarker of thrombosis in primary antiphospholipid syndrome. Arthritis Res Ther. 2019;21:10.

21. Tammaro A, Derive M, Gibot S, Leemans JC, Florquin S, Dessing MC. TREM-1 and its potential ligands in non-infectious diseases: from biology to clinical perspectives. Pharmacol Ther. 2017;177:81-95.

22. Gao S, Yi Y, Xia G, Yu C, Ye C, Tu F, Shen L, Wang W, Hua C. The characteristics and pivotal roles of triggering receptor expressed on myeloid cells-1 in autoimmune diseases. Autoimmun Rev. 2019;18:25-35.

23. Bleharski JR, Kiessler V, Buonsanti C, Sieling PA, Stenger S, Colonna M, Modlin RL. A role for triggering receptor expressed on myeloid cells-1 in host defense during the early-induced and adaptive phases of the immune response. J Immunol. 2003:170:3812-8.

24. Colonna M. TREMs in the immune system and beyond. Nat Rev Immunol. 2003:3:445-53.

25. Collins CE, La DT, Yang HT, Massin F, Gibot S, Faure G, Stohl W. Elevated synovial expression of triggering receptor expressed on myeloid cells 1 in patients with septic arthritis or rheumatoid arthritis. Ann Rheum Dis. 2009; 68:1768-74.

26. Kuai J, Gregory B, Hill A, Pittman DD, Feldman JL, Brown T, Carito B, O'Toole M, Ramsey R, Adolfsson O, et al. TREM-1 expression is increased in the synovium of rheumatoid arthritis patients and induces the expression of pro-inflammatory cytokines. Rheumatology (Oxford). 2009;48:1352-8

27. Bosco MC, Pierobon D, Blengio F, Raggi F, Vanni C, Gattorno M, Eva A, Novelli F, Cappello P, Giovarelli M, et al. Hypoxia modulates the gene expression profile of immunoregulatory receptors in human mature dendritic cells: identification of TREM-1 as a novel hypoxic marker in vitro and in vivo. Blood. 2011;117:2625-39.

28. Murakami Y, Akahoshi T, Aoki N, Toyomoto M, Miyasaka N, Kohsaka H. Intervention of an inflammation amplifier, triggering receptor expressed on myeloid cells 1, for treatment of autoimmune arthritis. Arthritis Rheum. 2009;60:1615-23.

29. Shen ZT, Sigalov AB. Rationally designed ligand-independent peptide inhibitors of TREM-1 ameliorate collagen-induced arthritis. J Cell Mol Med. 2017;21:2524-34.

30. Nah SS, Won HJ, Ha E, Kang I, Cho HY, Hur SJ, Lee SH, Baik HH. Epidermal growth factor increases prostaglandin E2 production via ERK1/2 MAPK and NF-kappaB pathway in fibroblast like synoviocytes from patients with rheumatoid arthritis. Rheumatol Int. 2010;30:443-9.

31. Murakami Y, Kohsaka H, Kitasato H, Akahoshi T. Lipopolysaccharide-induced up-regulation of triggering receptor expressed on myeloid cells-1 expression on macrophages is regulated by endogenous prostaglandin E2. J Immunol. 2007;178:1144-50.

32. Yuan Z, Mehta HJ, Mohammed K, Nasreen N, Roman R, Brantly M, Sadikot RT. TREM-1 is induced in tumor associated macrophages by cyclo- 
oxygenase pathway in human non-small cell lung cancer. PLoS One. 2014;9: e94241.

33. Roberts CA, Dickinson AK, Taams LS. The interplay between monocytes/ macrophages and CD4(+) T cell subsets in rheumatoid arthritis. Front Immunol. 2015;6:571.

34. Okada T, Tsukano H, Endo M, Tabata M, Miyata K, Kadomatsu T, Miyashita K, Semba K, Nakamura E, Tsukano M, et al. Synoviocyte-derived angiopoietinlike protein 2 contributes to synovial chronic inflammation in rheumatoid arthritis. Am J Pathol. 2010;176:2309-19.

35. Paulissen SM, van Hamburg JP, Davelaar N, Asmawidjaja PS, Hazes JM, Lubberts E. Synovial fibroblasts directly induce Th17 pathogenicity via the cyclooxygenase/prostaglandin E2 pathway, independent of IL-23. J Immunol. 2013;191:1364-72.

36. Yoon BR, Chung YH, Yoo SJ, Kawara K, Kim J, Yoo IS, Park CG, Kang SW, Lee WW. Preferential induction of the T cell auxiliary signaling molecule B7- $\mathrm{H} 3$ on synovial monocytes in rheumatoid arthritis. J Biol Chem. 2016;291:4048-57.

37. Rossol M, Kraus S, Pierer M, Baerwald C, Wagner U. The CD14(bright) CD16+ monocyte subset is expanded in rheumatoid arthritis and promotes expansion of the Th17 cell population. Arthritis Rheum. 2012;64:671-7.

38. Gibbons $L$, Hyrich KL. Biologic therapy for rheumatoid arthritis: clinical efficacy and predictors of response. Biodrugs. 2009;23:111-24.

39. Neidhart M, Gay RE, Gay S. Anti-interleukin-1 and anti-CD44 interventions producing significant inhibition of cartilage destruction in an in vitro model of cartilage invasion by rheumatoid arthritis synovial fibroblasts. Arthritis Rheum. 2000:43:1719-28.

40. Goldberg M, Nadiv O, Luknar-Gabor N, Agar G, Beer Y, Katz Y. Synergism between tumor necrosis factor alpha and interleukin-17 to induce IL-23 p19 expression in fibroblast-like synoviocytes. Mol Immunol. 2009;46:1854-9.

41. Demasi M, Cleland LG, Cook-Johnson RJ, James MJ. Effects of hypoxia on the expression and activity of cyclooxygenase 2 in fibroblast-like synoviocytes: interactions with monocyte-derived soluble mediators. Arthritis Rheum. 2004:50:2441-9.

42. Gomez-Pina V, Martinez E, Fernandez-Ruiz I, Del FC, Soares-Schanoski A, Jurado T, Siliceo M, Toledano V, Fernandez-Palomares R, Garcia-Rio F, et al. Role of MMPs in orchestrating inflammatory response in human monocytes via a TREM-1-PI3K-NF-kappaB pathway. J Leukoc Biol. 2012;91:933-45.

43. Ferat-Osorio E, Wong-Baeza I, Esquivel-Callejas N, Figueroa-Figueroa S, DuarteRojo A, Guzman-Valdivia-Gomez G, Rodea-Rosas H, Torres-Gonzalez R, SanchezFernandez $P$, Arriaga-Pizano $L$, et al. Triggering receptor expressed on myeloid cells-1 expression on monocytes is associated with inflammation but not with infection in acute pancreatitis. Crit Care. 2009;13:R69.

44. Cavaillon JM. Monocyte TREM-1 membrane expression in non-infectious inflammation. Crit Care. 2009:13:152

45. Carrasco K, Boufenzer A, Jolly L, Le Cordier H, Wang G, Heck AJ, Cerwenka A, Vinolo E, Nazabal A, Kriznik A, et al. TREM-1 multimerization is essential for its activation on monocytes and neutrophils. Cell Mol Immunol. 2019;16: 460-72.

46. Syed MA, Joo M, Abbas Z, Rodger D, Christman JW, Mehta D, Sadikot RT. Expression of TREM-1 is inhibited by PGD2 and PGJ2 in macrophages. Exp Cell Res. 2010;316:3140-9.

47. Amin AR, Attur M, Abramson SB. Nitric oxide synthase and cyclooxygenases: distribution, regulation, and intervention in arthritis. Curr Opin Rheumatol. 1999;11:202-9.

48. Crofford LJ, Lipsky PE, Brooks P, Abramson SB, Simon LS, van de Putte LB. Basic biology and clinical application of specific cyclooxygenase-2 inhibitors. Arthritis Rheum. 2000;43:4-13.

49. Peng $L$, Zhou Y, Dong L, Chen RQ, Sun GY, Liu T, Ran WZ, Fang X, Jiang JX, Guan CX. TGF-beta1 upregulates the expression of triggering receptor expressed on myeloid cells 1 in murine lungs. Sci Rep. 2016;6:18946.

50. Mihailidou I, Pelekanou A, Pistiki A, Spyridaki A, Tzepi IM, Damoraki G, Giamarellos-Bourboulis EJ. Dexamethasone down-regulates expression of triggering receptor expressed on myeloid Cells-1: evidence for a TNFalpharelated effect. Front Public Health. 2013;1:50

51. Rao VH, Rai V, Stoupa S, Subramanian S, Agrawal DK. Data on TREM-1 activation destabilizing carotid plaques. Data Brief. 2016;8:230-4.

52. Murakami Y, Akahoshi T, Hayashi I, Endo H, Kawai S, Inoue M, Kondo H, Kitasato $\mathrm{H}$. Induction of triggering receptor expressed on myeloid cells 1 in murine resident peritoneal macrophages by monosodium urate monohydrate crystals. Arthritis Rheum. 2006;54:455-62.

53. Stahl HD, Hubner B, Seidl B, Liebert UG, van der Heijden IM, Wilbrink B, Kraan MC, Emmrich F, Tak PP. Detection of multiple viral DNA species in synovial tissue and fluid of patients with early arthritis. Ann Rheum Dis. 2000;59:342-6.

54. Piccinini AM, Midwood KS. DAMPening inflammation by modulating TLR signalling. Mediat Inflamm. 2010;2010:672395.

55. Zheng H, Heiderscheidt CA, Joo M, Gao X, Knezevic N, Mehta D, Sadikot RT. MYD88-dependent and -independent activation of TREM-1 via specific TLR ligands. Eur J Immunol. 2010;40:162-71.

56. Campanholle G, Mittelsteadt K, Nakagawa S, Kobayashi A, Lin SL, Gharib SA, Heinecke JW, Hamerman JA, Altemeier WA, Duffield JS. TLR-2/TLR-4 TREM-1 signaling pathway is dispensable in inflammatory myeloid cells during sterile kidney injury. PLoS One. 2013:8:e68640.

\section{Publisher's Note}

Springer Nature remains neutral with regard to jurisdictional claims in published maps and institutional affiliations.
Ready to submit your research? Choose BMC and benefit from:

- fast, convenient online submission

- thorough peer review by experienced researchers in your field

- rapid publication on acceptance

- support for research data, including large and complex data types

- gold Open Access which fosters wider collaboration and increased citations

- maximum visibility for your research: over $100 \mathrm{M}$ website views per year

At $\mathrm{BMC}$, research is always in progress.

Learn more biomedcentral.com/submissions 